\title{
Confinement Facilitated Protein Stabilization As Investigated by Small-Angle Neutron Scattering
}

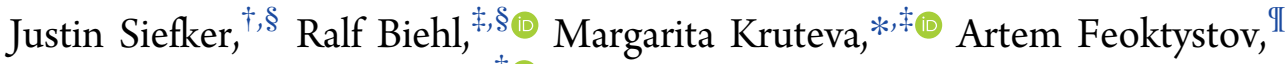 \\ and Marc-Olivier Coppens* $*$
}

${ }^{\dagger}$ Centre for Nature Inspired Engineering (CNIE) and Department of Chemical Engineering, University College London, London WC1E 7JE, United Kingdom

${ }^{\ddagger}$ Jülich Centre for Neutron Science (JCNS-1) and Institute for Complex Systems (ICS-1), Forschungszentrum Jülich GmbH, Jülich 52425, Germany

IJülich Centre for Neutron Science (JCNS) at Heinz Maier-Leibnitz Zentrum (MLZ), Forschungszentrum Jülich GmbH, Garching 85747, Germany

Supporting Information

\begin{abstract}
While mesoporous silicas have been shown to be a compelling candidate for drug delivery and the implementation of biotechnological applications requiring protein confinement and immobilization, the understanding of protein behavior upon physical adsorption into silica pores is limited. Many indirect methods are available to assess general adsorbed protein stability, such as Fourier-transform infrared spectroscopy and activity assays. However, the limitation of these methods is that spatial protein arrangement within the pores cannot be assessed. Mesoporous silicas pose a distinct challenge to direct methods, such as transmission electron microscopy, which lacks the contrast and resolution required to adequately observe immobilized protein structure, and nuclear magnetic resonance, which is computationally intensive and requires knowledge of the primary structure a priori. Small-angle neutron scattering can surmount these limitations and observe spatial protein arrangement within pores. Hereby, we observe the stabilization of fluidlike protein arrangement, facilitated by geometry-dependent crowding effects in cylindrical pores of ordered mesoporous silica, SBA-15. Stabilization is induced from a fluid-like structure factor, which is observed for samples at maximum protein loading in SBA-15 with pore diameters of 6.4 and $8.1 \mathrm{~nm}$. Application of this effect for prevention of irreversible aggregation in high concentration environments is proposed.
\end{abstract}

$\mathrm{C}$ onfinement of biomolecules in structured nanoporous materials offers advantages, such as improved stability, in biological and synthetic systems. Immobilization of proteins on confined surfaces is essential to many applications of modern protein engineering in areas of catalysis, sensing, separations, and medicine. ${ }^{1-3}$ In confined environments, geometric properties, such as surface curvature, play a significant role in addition to physical properties. Ordered mesoporous materials like Santa Barbara Amorphous-15 (SBA-15) silica are considered good candidates for these applications, due to their large pore volume and surface area, as well as tunable pore size, geometry, and surface chemistry. Two main challenges of protein confinement are (i) loading of protein into the mesoporous structure (immobilization, pore packing efficiency), and (ii) influence of surface interactions on protein structure and dynamics.

Under certain conditions, pore filling limitations can be overcome and the maximum theoretical material packing limit can be reached. ${ }^{4}$ Sang and Coppens have shown how pore surface geometry and chemistry affect activity and structure of adsorbed protein. ${ }^{5}$ The authors observed enhanced enzymatic activity when protein was electrostatically adsorbed in cylindrical pores of a diameter barely wider than the protein, and that this enhancement effect depends on the degree of pore curvature. The activity increase also correlated with preservation of protein secondary structure, as quantified by FTIR. Tertiary structural changes could only be presumed, leaving additional structural and dynamic details to be desired. Elastic and quasielastic neutron scattering of myoglobin $(\mathrm{mb})$ in silica hydrogel revealed the crucial role porous silica matrices can play, ${ }^{6}$ and SBA-15 has been discussed as a compelling candidate for immobilization and delivery of protein therapeutics. ${ }^{7}$ The uniqueness of SBA-15 derives from similarity to the GroEL/ES chaperonin, which assists the proper folding and refolding processes of small, cytosolic, globular proteins in the aggregation prone cellular environment. ${ }^{8}$ This chaperonin facilitates folding via a kinetically mediated minimization of free energy from an initially perturbed, destabilized state. As in SBA-15, steric and electrostatic force balancing effects are crucial.

SBA-15 has well-defined geometric properties with conveniently characterizable defects that allow for ease in modeling and experimental characterization. ${ }^{9}$ Locally cylindrical pores enable structural analysis, and control of surface curvature. $^{10}$ Thus, SBA-15 is the primary material for investigation, alongside the topological variant KIT-6 (Korean Advanced Institute of Science and Technology-6; an $I a \overline{3} d$ bigyroidal, body-centered cubic structure with twisted, interpenetrating, bicontinuous, constant-diameter pores), allowing

Received: August 7, 2018

Published: September 27, 2018 
investigation of compelling materials, while enabling robust analysis. Herein, we report on adsorbed protein arrangement in pores with respect to confining pore morphology and adsorbed protein concentration, for lysozyme (lyz) and myoglobin $(\mathrm{mb})$, informed by small-angle neutron scattering (SANS). This study observes the stabilization of fluid-like proteinprotein coordination of lysozyme and myoglobin driven by intrinsically high protein pore concentration and nanoporous confining geometry. The experimental space of this investigation is shown in Figure 1.

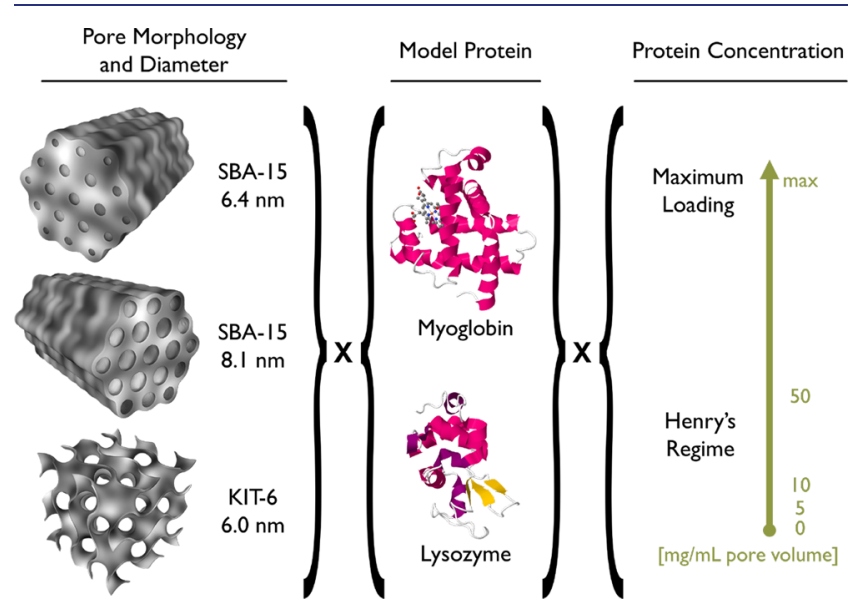

Figure 1. Experimental sample space. To better illustrate geometry, the KIT- 6 representation portrays an exaggerated pore diameter to unit cell size ratio.

The materials, proteins, and concentrations chosen allow comparison over confinement curvature, pore topology, protein type and charge, and concentration regime. Samples were evaluated in $25 \mathrm{mM}$ phosphate $\mathrm{D}_{2} \mathrm{O}$ buffer $(\mathrm{pH} 7.2)$ within the activity range of each protein ensuring structural integrity. ${ }^{11,12}$ This results in a net neutral charge of myoglobin (pI 7.2), and a net positive charge of lysozyme (pI 11.35), facilitating electrostatic attraction with the silica surface (pI 2). Protein concentrations of $5,10,50 \mathrm{mg} / \mathrm{mL}$ pore volume, and maximum pore loading (see Supporting Information [SI]) were selected to evaluate both Henry's regime (minimal protein-protein interactions) and concentration with greatest protein-protein interaction. Sample preparation is discussed in the SI and includes hydrogen/deuterium (H/D) exchange, protein physisorption, and sedimentation into analysis cells as an isotropic powder. SANS measurements were performed using the KWS-1 instrument operated by Jülich Centre for Neutron Science (JCNS) at the Heinz Maier-Leibnitz Zentrum (MLZ).

To determine the protein shape observed by SANS and examine protein-protein interactions, bulk protein samples were measured (see SI). Protein size and shape was evaluated using an ellipsoid of revolution form factor. ${ }^{13}$ This resulted in a volume of $18.2 \pm 0.9 \mathrm{~nm}^{3}$ and axial ratio of 0.43 for myoglobin (oblate ellipsoid), and a volume of $17.8 \pm 0.3 \mathrm{~nm}^{3}$ and axial ratio of 2.3 for lysozyme (prolate ellipsoid). At low concentrations, form factors show agreement with Protein Data Bank crystal structures (PDB IDs: 2lyz and $1 \mathrm{mbn}$ ). Above $50 \mathrm{mg} / \mathrm{mL}$, the observed myoglobin structure factor suggests repulsion, while the lysozyme structure factor suggests a partially attractive interaction. Bulk samples of maximum protein concentration for both proteins show a loss of monomeric concentration, due to sedimentation of protein aggregates. This effect is more pronounced for lysozyme, due to attractive interactions.

Unloaded material profiles were modeled using a scattering intensity expression described by Engel et al. ${ }^{14}$ and complemented by convolution with the resolution function of the instrument according to procedures described by Pedersen et al. ${ }^{15}$ (see Figure 2 and SI).

$$
I(q)=I_{\mathrm{P}}(q)+K \mathrm{e}^{-\sigma_{\mathrm{DW}}{ }^{2}{ }^{2}} S_{\mathrm{SiO}_{2}}(q)\left\langle F_{\mathrm{c}}(q)\right\rangle^{2}+I_{\mathrm{diff}}(q)+I_{\mathrm{inc}}
$$

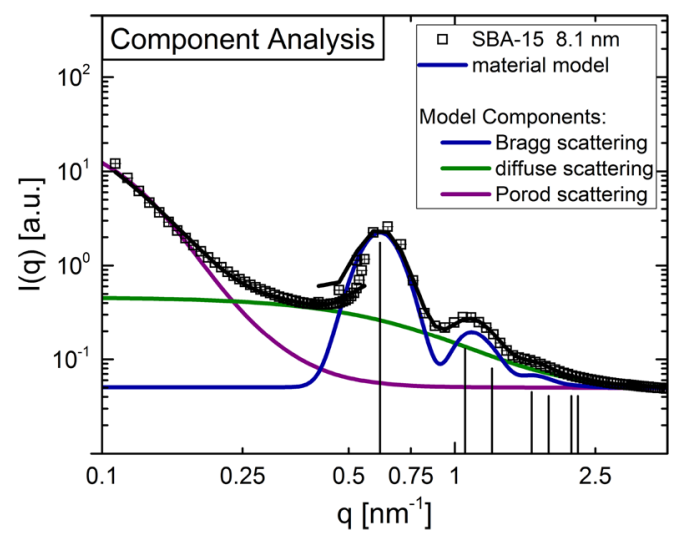

Figure 2. SBA-15 $8.1 \mathrm{~nm}$ material scattering and model fit. Each component includes the incoherent background contribution: Bragg reflections, diffuse scattering, and Porod scattering (each scaled to best fit). Bragg reflection peaks are marked with vertical lines (listed in sequence: (100), (110), (200), (210), (300), (220), (310)). All contributions are smeared by the resolution of the instrument resulting in a break at approximately $0.4 \mathrm{~nm}^{-1}$, due to a change in detector distance.

The space group geometry of the oriented pore lattice is described by a material structure factor, $S_{\mathrm{SiO}_{2}}(q)$, and cylindrical form factor, $\left\langle F_{c}(q)\right\rangle^{2}$, including pore size distribution (25\% width). Bragg scattering peaks are described by Gaussian functions; due to an intrinsically similar nature, pore size distribution, pore wall roughness, and lattice distortions lead to a gradual decrease of Bragg peak intensities described by a Debye-Waller like factor, $\mathrm{e}^{-\sigma_{\mathrm{DW}} q^{2}}$. This preferentially suppresses the signal from higher order Bragg peaks. ${ }^{16}$ Diffuse scattering, $I_{\text {diff }}(q)$, is due to material distortions, such as lattice distortions or material inhomogeneity, and includes deviation from a cylindrical pore geometry. ${ }^{17} \mathrm{Eq} 1$ accounts for Porod scattering, $I_{\mathrm{P}}(q), \sim q^{-4}$, from the particle surface, and incoherent background, $I_{\text {inc }}$. As seen in Figure 2, this allows for a well conditioned fit of the materials, in good agreement with supporting characterization data (see SI). In particular, for $q$-values greater than the first Bragg peak, the main contribution to intensity is from diffuse scattering.

Figure 3 shows the scattering intensities for SBA-15 with 6.4 and $8.1 \mathrm{~nm}$ pores loaded with myoglobin and lysozyme. While lower loadings show general similarity over scattering profiles (see SI for KIT-6 $6.0 \mathrm{~nm}$ ), a distinct difference for the highest concentration samples (maximal loadings) becomes apparent for SBA-15 $8.1 \mathrm{~nm}$. At maximal loadings, a strongly broadened peak between 1.5 and $2.5 \mathrm{~nm}^{-1}$ appears. Concurrently, the second-order hexagonal peaks (110) and (200) vanish, which is also found for the $6.4 \mathrm{~nm}$ sample. Otherwise, it is observed 


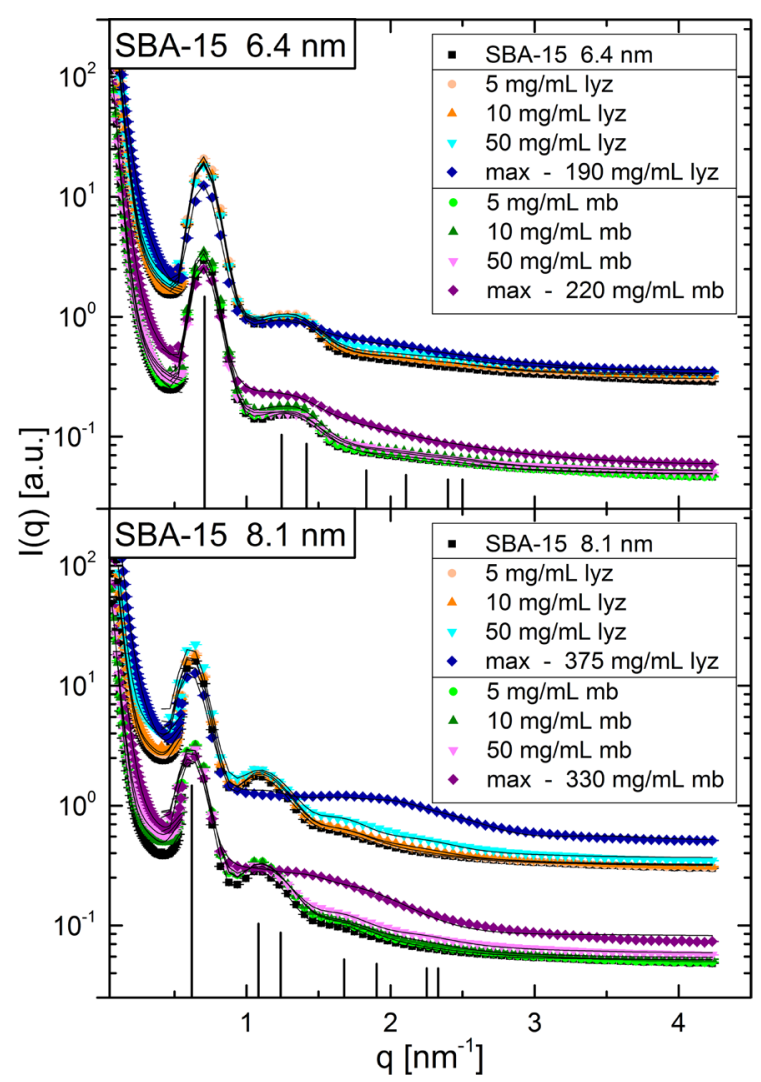

Figure 3. Scattering of myoglobin and lysozyme loaded on SBA-15 with a mesopore diameter of 6.4 and $8.1 \mathrm{~nm}$. Lysozyme data are shifted by a factor of 10 . Black lines show the material model, which is complemented by an additional protein contribution for maximal protein concentration samples $(\max )$. Protein contributions are incorporated using the fluid-like 3D Percus-Yevick structure factor. Bragg reflection peaks are marked with vertical lines (listed in sequence: (100), (110), (200), (210), (300), (220), (310)). All contributions are smeared by the resolution of the instrument resulting in a break at approximately $0.4 \mathrm{~nm}^{-1}$ due to a change in detector distance.

that with increasing protein concentration, the first hexagonal peak (100) is flanked by a larger surrounding background, and that, at high $q$-values, general scattering intensity increases with protein concentration.

When evaluating lower loading concentrations ( $\leq 50 \mathrm{mg} /$ $\mathrm{mL}$ ) and KIT-6 samples, low signal amplitude arising from additional protein does not allow direct extraction of reliable protein information. Protein adsorbed onto the wall or free inside the pores is regarded as a distortion of the scattering length density distribution and contributes to the diffuse scattering term. ${ }^{18}$ This results in a rise of the diffuse scattering amplitude observable as an increase in intensity below the first peak in Figure 3. Only a minor change is observed in the corresponding correlation length, $\xi_{\text {diff }}$ (see SI). The scattering contrast of protein $\left(4 \times 10^{-4} \mathrm{~nm}^{-2}\right)$ is only larger than the scattering contrast of silica $\left(2.1 \times 10^{-4} \mathrm{~nm}^{-2}\right)$ by a factor of 2 (see SI). Therefore, scattering contributions attributed to protein could also be interpreted as resulting from the pore size distribution, pore wall microporosity, pore surface roughness, and lattice distortions, all of which lead to a lower DebyeWaller like factor (increased $\sigma_{\mathrm{DW}}$ ). In general, higher-order peaks of the SBA-15 porous lattices vanish as $\sigma_{\mathrm{DW}}$ increases

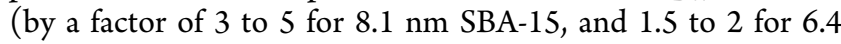

nm SBA-15). Similar behavior is observed for KIT-6 samples, although the second-order peak shape is distorted by the variable pore radius or curvature. Nonetheless, the first peak is well described by a cylindrical pore geometry, independently of KIT-6 topology. The average contrast between the silica matrix and solvent decreases with increasing protein concentration, reducing the observed (100) peak intensity. This conclusion is supported by the lesser degree in which these effects are present for the KIT-6 samples, as this material adsorbs significantly less protein per pore volume (see SI).

Additional intensity observed for maximum loaded SBA-15 samples cannot be explained by diffuse scattering or by incorporating protein form factor scattering. These components, at adequate protein concentrations, cannot reproduce the broad peaks observed. Alternatively, the high packing density with direct protein interactions could promote a fluidlike spatial arrangement within the pores, which can be represented by a fluid-like structure factor, such as the PercusYevick structure factor. ${ }^{19,20}$ The scattering intensity of a fluid arrangement of proteins is described as the product of its structure and form factors. Restriction by nanoconfinement in cylindrical pores, however, changes the observable form factor to the convolution $(*)$ of the cylinder form factor with the protein form factor (see SI for derivation). This leads to an additional contribution to the diffuse scattering term, as

$$
I_{\text {diff, } \mathrm{P}}(q)=n_{\mathrm{C}} n_{\mathrm{P}}\left|\left\langle C(q) * \mathrm{Q}_{\mathrm{p}}(q)\right\rangle_{\mathrm{eP}}\right|^{2} S_{\mathrm{P}}(q)
$$

which depends on cylinder density, $n_{\mathrm{C}}$, protein density, $n_{\mathrm{P}}$, protein scattering amplitude, $\varrho_{\mathrm{p}}(q)$ (yielding the protein form factor as $\left.\left\langle\varrho_{\mathrm{P}}(q) \varrho_{\mathrm{P}}^{*}(q)\right\rangle\right)$, cylinder scattering amplitude, $C(q)$, and fluid structure factor, $S_{\mathrm{P}}(q)$.

Scattering intensities of samples loaded to saturation are fit with eq 1 , incorporating the diffuse scattering contribution of eq 2. Protein is described as an ellipsoid of revolution equivalent to bulk protein. The Percus-Yevick structure factor $^{19,20}$ is used as an approximation for the real structure factor between asymmetric ellipsoids, assuming broader distribution of volume fractions in the pores (Gaussian, cut at the upper one-sigma border). Following this hypothesis, volume fractions of approximately $64 \%$ for lysozyme and $40 \%$ for myoglobin are found. The preferential adsorption of lysozyme compared to myoglobin has been observed before. ${ }^{21}$ Such high volume fractions indicate the Percus-Yevick structure factor may only serve as an approximation of the real protein-protein structure. The maximum volume fraction for disordered close packed spheres is $64 \%$ and for hexagonal close packing is $74 \%$. For lysozyme the disordered close packing limit is reached. Reproduction of the observed peak positions corroborates a very close fluid-like packing of the proteins. As proteins are not completely rigid spherical objects, even higher packing fractions are potentially achievable, while still maintaining a fluid-like structure.

It is remarkable that lysozyme, with the higher packing fraction and attractive protein-protein interactions, is not extensively aggregated. One explanation could be that lysozyme creates disordered pore blocking aggregates within the pore matrix, although prior research shows increased enzymatic activity under these sample conditions. ${ }^{5}$ Alternatively, this result suggests the confining environment and geometry inhibit undesirable protein-protein interactions that prevent protein deactivation and stabilize protein packing at high concentrations. The moderate volume fraction of 
myoglobin (40\%) allows for protein movement, suggesting characteristically nonsterically hindered fluid-like behavior.

For both cases, it is striking that neither show power law scattering $\sim q^{-d_{\mathrm{f}}}$, with fractal dimension $d_{\mathrm{f}}$, which is associated with formation of aggregates or aggregated clusters. ${ }^{22}$ This is remarkable, because both can form aggregates or fibrils at such high concentrations. ${ }^{23-25}$ While myoglobin stabilization may be due to the heme group, unconfined lysozyme would be expected to aggregate at such high concentration and surface proximity. One possibility is that, due to attractive electrostatic interactions from the silica surface and adjacent nearest neighbor protein pairs, lysozyme monomers are not frozen, but stabilized in a high-density, fluid-like state. As narrow, cylindrical pores limit protein-protein interactions to be quasione-dimensional, cylindrical mesopores could prevent irreversible aggregation by sterically hindering high-mer aggregate formation, and only allowing formation of dynamically reversible low-mer lysozyme aggregates. ${ }^{26}$ This is supported by the fact that the partially unfolded state of lysozyme, which forms dimer aggregates, is fully reversible, considered an inherent fluctuation of the native protein state, and on the native side of the free energy barrier of unfolding. ${ }^{26}$ This effect, in conjunction with steric restrictions, limits formation of amyloid fibrils (which require unfolding of the protein monomer) and amyloid-like protofibrils (which require highmer protein aggregates). Because of the electrostatic interactions, confinement could also facilitate the reversibility of low-mer aggregate formation. As this environment bears similarities to the GroEL/ES chaperonin, ${ }^{7}$ and induced protection effects have been previously demonstrated, ${ }^{27}$ this proposition is compelling.

To the authors' knowledge, this is the first time confinement-facilitated protein arrangement has been observed. The analysis and observations were enabled through the use of the well-defined silica, SBA-15, and the use of SANS, under conditions allowing sufficient protein-material scattering contrast. This work demonstrates how SANS offers opportunities to observe the spatial order of proteins confined within ordered substrates and can drive insights about confinement behavior.

\section{ASSOCIATED CONTENT}

\section{S Supporting Information}

The Supporting Information is available free of charge on the ACS Publications website at DOI: 10.1021/jacs.8b08454.

Scattering models, model specification, protein characterization, and material characterization (PDF)

\section{AUTHOR INFORMATION}

\section{Corresponding Authors}

*M.Kruteva@fz-juelich.de

*M.Coppens@ucl.ac.uk

\section{ORCID}

Ralf Biehl: 0000-0002-1999-547X

Margarita Kruteva: 0000-0002-7686-0934

Marc-Olivier Coppens: 0000-0002-1810-2537

\section{Author Contributions}

${ }^{\S}$ Authors contributed equally to this work.

Notes

The authors declare no competing financial interest.

\section{ACKNOWLEDGMENTS}

This work was completed with support from the EPSRC "Frontier Engineering" Centre for Nature Inspired Engineering (via EP/K038656/1), and is based upon experiments performed at the KWS-1 instrument (proposals no. 9593 and 10018) operated by the Jülich Centre for Neutron Science at the Heinz Maier-Leibnitz Zentrum (MLZ) located in Garching, Germany.

\section{REFERENCES}

(1) Luckarift, H. R.; Spain, J. C.; Naik, R. R.; Stone, M. O. Nat. Biotechnol. 2004, 22, 211-213.

(2) Menaa, B.; Torres, C.; Herrero, M.; Rives, V.; Gilbert, A. R.; Eggers, D. K. Biophys. J. 2008, 95, L51-L53.

(3) Angenendt, P. Drug Discovery Today 2005, 10, 503-511.

(4) Sang, L.-C.; Vinu, A.; Coppens, M.-O. Langmuir 2011, 27, $13828-13837$.

(5) Sang, L.-C.; Coppens, M.-O. Phys. Chem. Chem. Phys. 2011, 13, 6689.

(6) Schirò, G.; Sclafani, M.; Caronna, C.; Natali, F.; Plazanet, M.; Cupane, A. Chem. Phys. 2008, 345, 259-266.

(7) Siefker, J.; Karande, P.; Coppens, M.-O. Expert Opin. Drug Delivery 2014, 11, 1781-1793.

(8) Hayer-Hartl, M.; Bracher, A.; Hartl, F. U. Trends Biochem. Sci. 2016, 41, 62-76.

(9) Hofmann, T.; Wallacher, D.; Perlich, J.; Koyiloth Vayalil, S.; Huber, P. Langmuir 2016, 32, 2928-2936 PMID: 26940230 .

(10) Shin, T.; Findenegg, G. H.; Brandt, A. In Smart Colloidal Materials; Richtering, W., Ed.; Springer-Verlag: Berlin/Heidelberg, 2006; Vol. 133; pp 116-122.

(11) Carlsen, C. U.; Skovgaard, I. M.; Skibsted, L. H. J. Agric. Food Chem. 2003, 51, 5815-5823.

(12) Carroll, S. F.; Martinez, R. J. Infect. Immun. 1979, 24, 460-467.

(13) Pedersen, J. S. Adv. Colloid Interface Sci. 1997, 70, 171-210.

(14) Engel, M.; Stühn, B.; Schneider, J. J.; Cornelius, T.; Naumann, M. Appl. Phys. A: Mater. Sci. Process. 2009, 97, 99-108.

(15) Pedersen, J. S.; Posselt, D.; Mortensen, K. J. Appl. Crystallogr. 1990, 23, 321-333.

(16) Förster, S.; Timmann, A.; Konrad, M.; Schellbach, C.; Meyer, A.; Funari, S. S.; Mulvaney, P.; Knott, R. J. Phys. Chem. B 2005, 109, $1347-1360$.

(17) Shin, T. G.; Müter, D.; Meissner, J.; Paris, O.; Findenegg, G. H. Langmuir 2011, 27, 5252-5263.

(18) Müter, D.; Shin, T.; Demé, B.; Fratzl, P.; Paris, O.; Findenegg, G. H. J. Phys. Chem. Lett. 2010, 1, 1442-1446.

(19) Wertheim, M. S. Phys. Rev. Lett. 1963, 10, 321.

(20) Percus, J. K.; Yevick, G. J. Phys. Rev. 1958, 110, 1.

(21) Moerz, S. T.; Huber, P. J. Phys. Chem. C 2015, 119, 2707227079.

(22) Sorensen, C. M. Aerosol Sci. Technol. 2011, 45, 765-779.

(23) Iannuzzi, C.; Maritato, R.; Irace, G.; Sirangelo, I. Int. J. Mol. Sci. 2013, 14, 14287-14300.

(24) Chiti, F.; Dobson, C. M. Nat. Chem. Biol. 2009, 5, 15-22.

(25) Dumoulin, M.; Kumita, J. R.; Dobson, C. M. Acc. Chem. Res. 2006, 39, 603-610.

(26) Canet, D.; Last, A. M.; Tito, P.; Sunde, M.; Spencer, A.; Archer, D. B.; Redfield, C.; Robinson, C. V.; Dobson, C. M. Nat. Struct. Biol. 2002, 9, 308-315.

(27) Lynch, M. M.; Liu, J.; Nigra, M.; Coppens, M.-O. Langmuir 2016, 32, 9604-9610. 